\title{
Series Loss-Free Resistor: Analysis, Realization and Applications
}

\author{
Ivo Barbi, Life Fellow, IEEE
}

\begin{abstract}
An analysis of the transient behavior of DC networks consisting of voltage sources, capacitors, and series loss-free resistors (SLFRs) is presented. The realization of an SLFR was achieved by means of a topological variation of the positive outputvoltage buck-boost converter operated in discontinuous-conduction mode (DCM). It is demonstrated that even simple $\mathrm{RC}$ circuits containing SLFRs exhibit behavior that is described by Abel nonlinear differential equations, which do not have an exact analytical solution. Some possible applications of the SLFRs in power electronics, DC microgrids and renewable energy power systems, include loss-free charge and discharge of capacitor banks, voltage equalization of capacitors, and loss-free voltage clamping circuits. The concept of the SLFR can also be applied for voltage control in DC microgrids, employing the droop voltage technique, equalization of power and current in voltage sources associated in parallel (including batteries and DC-DC converters), and also input and output voltage natural balance in input-series output-series (ISOS) association of DC converters. The theoretical analysis results are validated via numerical examples and computer simulation.
\end{abstract}

Index Terms-Loss-free resistor (LFR), series loss-free resistor (SLFR), series loss-free resistor applications, transient analysis of circuits containing SLFR.

\section{INTRODUCTION}

The concept of the loss-free resistor (LFR) was introduced by Singer [1,2] and modeled by Singer and Erickson [3] using an equivalent two-port network, as shown in Fig. 1.

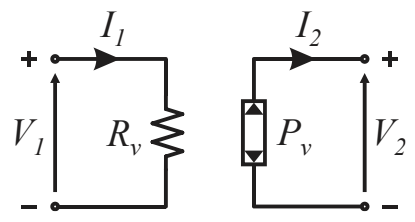

Fig. 1. Two-port equivalent circuit of the loss-free resistor.

The equivalent circuit exhibits a purely resistive behavior at the input, represented by the resistor $R_{v}$, and a power source $P_{v}$ at the output.

The LFR can be realized naturally with the use of some DCDC converters, such as the buck-boost, sepic, zeta and Cuk converters, with PWM modulation, operating in the discontinuous conduction mode (DCM) [3,4]. It is also common to realize the LFR with DC-DC converters operating in continuous conduction mode (CCM), with the use of closed-loop

I. Barbi is with the Electrical and Electronic Engineering Department, Federal University of Santa Catarina, Florianópolis, SC Brazil, (e-mail: ivobarbi@gmail.com). control to impose the input current proportional to the input voltage [6-8].

The most common use of LFR performed by DC-DC converters operating in both DCM and CCM is rectification with a unity power factor in the pre-regulating stages of power supplies [4-7]. The LFR concept was also adopted in the realization of voltage sources with regulated output without internal losses [8] and as a power interface for implementing MPPT in photovoltaic generators [9].

In all of the above-mentioned applications, the LFR is connected in parallel with the input power supply and all of the processed power is transferred to the load connected at the output terminals. An exception is the application described in [8], where the LFR is connected in series with the power supply and the load, and its absorbed energy is returned to the input voltage source.

This paper reports a study on the natural realization of a series loss-free resistor (SLFR). Some possible practical applications are discussed, and electrical transients in DC circuits formed by voltage sources, capacitors and SLFR are analyzed.

\section{SERIES LOSS-FREE RESISTOR (SLFR) AND ITS REALIZATION}

The loss-free resistor (LFR) represented by the two-port network shown in Fig. 1 is described by [4]

$$
\begin{aligned}
& V_{1}=R_{v} I_{1} \\
& P_{v}=R_{v} I_{1}^{2}
\end{aligned}
$$

As mentioned in the previous section, some converters when operating in DCM exhibit a natural (without control) loss-free resistor behavior. The simplest topology with this feature is the buck-boost or flyback converter, as shown in Fig. 2 [3].

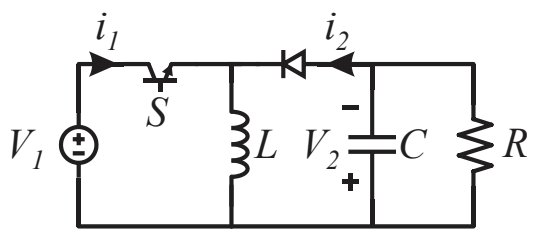

(a)

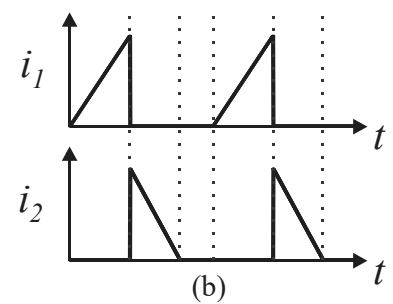

Fig. 2. Discontinuous conduction mode buck-boost converter: (a) power stage diagram and (b) input and output current waveforms. 
When the capacitor $\mathrm{C}$ is sufficiently large that its voltage ripple can be neglected, the converter operates in DCM mode if

$$
\frac{V_{2}}{V_{1}} \geq \frac{D}{1-D}
$$

The resistance value of the LFR is given by

$$
R_{v} \geq \frac{2 L f_{s}}{D^{2}}
$$

where $f_{s}$ denotes the switching frequency and $D$ denotes the duty-cycle [3]. The averaged equivalent circuit in terms of the LFR is shown in Fig. 3.

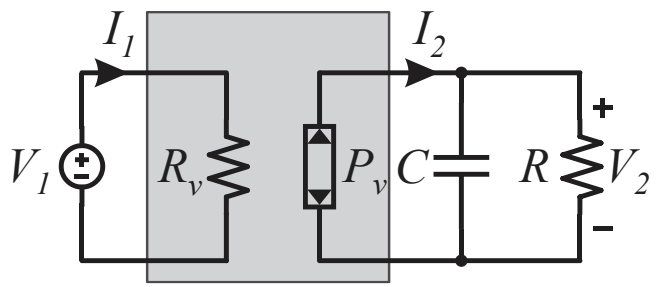

Fig. 3. Averaged equivalent circuit of the buck-converter operated in DCM, represented by the parallel LFR.

Although it is a simple topology with few components in its power stage, the buck-boost converter shown in Fig. 2 generates an output voltage with the polarity reversed in relation to the polarity of the input voltage, which may be undesirable in some applications.

This difficulty can be overcome with the use of the buck-boost converter with positive output voltage shown in Fig. 4, in which the two switches are gated on and off by the same signal. The waveforms of the input and output currents are the same as those of the negative output converter and the expressions (1), (2), (3) and (4) are valid for both converters. The equivalent circuit employing the LFR is the same as that shown in Fig. 3.

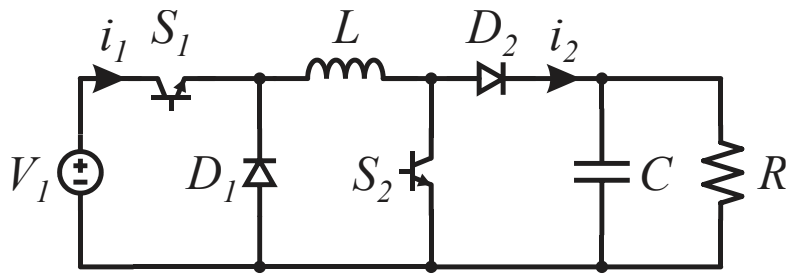

(a)

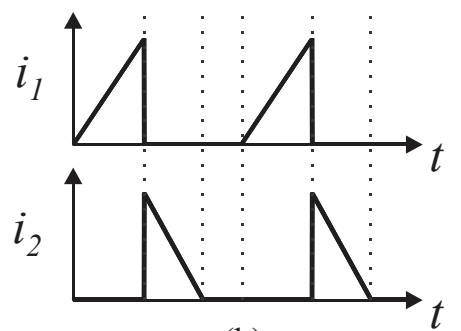

(b)

Fig. 4. Discontinuous conduction mode, positive output, buck-boost converter: (a) power stage diagram and, (b) input and output current waveforms.

\section{A. Equivalent Circuit of the SLFR and its Realization}

In the equivalent circuit shown in Fig. 3, which represents the converters shown in Figs. 2 and 4, the loss-free resistor is connected in parallel with the power supply [3].

In this section we describe the realization of the loss-free resistor connected in series with the input power source, whose equivalent circuit is shown in Fig. 5, in which the power source $P_{v}$ transfers all of the power absorbed by the resistor $R_{v}$ to the input voltage source $V_{1}$.

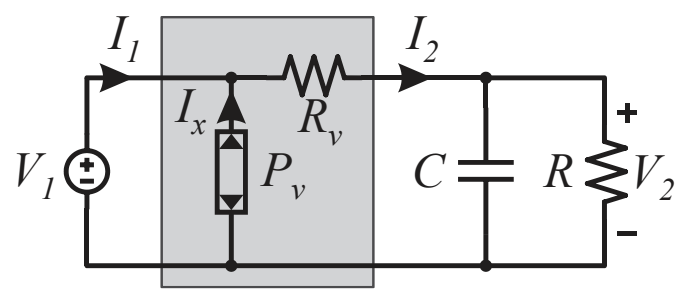

Fig. 5. Representation of the series loss-free resistor (SLFR).

The power absorbed by the loss-free resistor $R_{v}$ is given by

$$
P_{v}=\frac{\left(V_{1}-V_{2}\right)^{2}}{R_{v}}
$$

Hence, the current of the power source connected in parallel with the input voltage source $V_{1}$ is determined by

$$
I_{x}=\frac{\left(V_{1}-V_{2}\right)^{2}}{R_{v} V_{1}}
$$

The behavior of the circuit operating in steady-state is described by

$$
V_{2}=V_{1}-R_{v} I_{2}
$$

and

$$
\frac{V_{2}}{V_{1}}=\frac{R_{2}}{R_{2}+R_{v}}
$$

Expression (8) shows that it is possible to control the output voltage $V_{2}$ with no internal losses, through the appropriate adjustment of the value of $R_{v}$ [8].

It is not possible to realize the SLFR using the conventional buck-boost converter, due to the inversion of the polarity of the output voltage in relation to the input voltage. However, this realization is possible with the use of the topological variation of the flyback converter, as shown in Fig. 6.

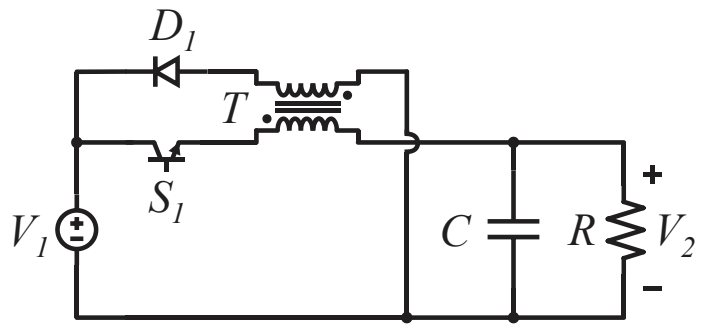

Fig. 6. Realization of the SLFR using a topological variation of the flyback converter operating in DCM. 
The transformer can be avoided by using the topological variation of the buck-boost converter with positive output voltage, to obtain the SLFR, as shown in Fig. 7.

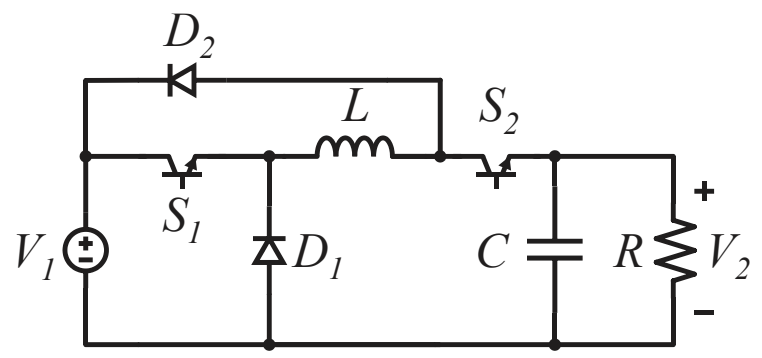

Fig. 7. Realization of the SLFR using a topological variation of the positive output voltage buck-boost converter operating in DCM.

\section{B. Operation Principle of the Proposed Circuit}

The topological stages of the ideal converter operating in DCM for the natural realization of the SLFR are shown in Fig. 8 and the theoretical relevant current waveforms associated with the circuit are shown in Fig. 9.

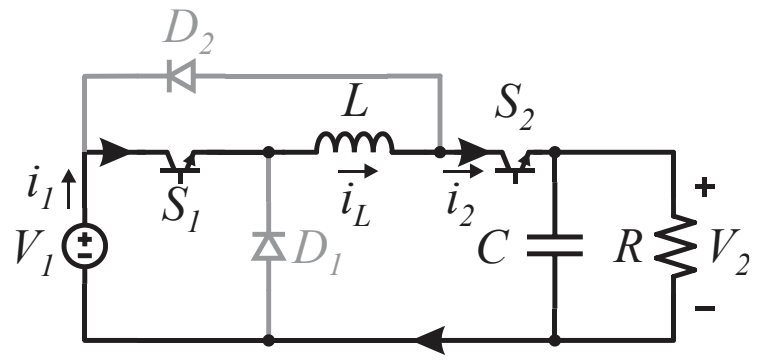

(a)

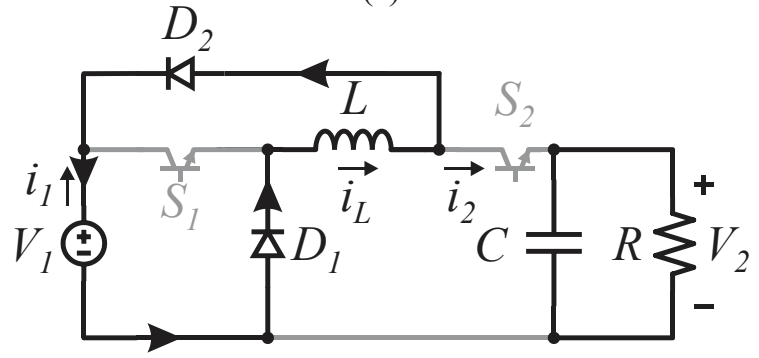

(b)

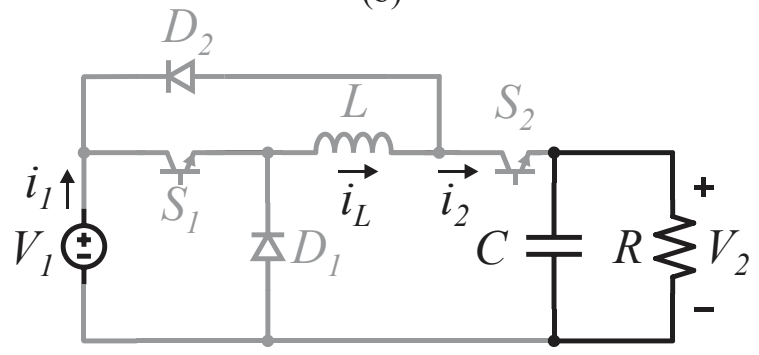

(c)

Fig. 8. Current-flow paths of the proposed converter in DCM. (a) Time interval ( $\left.0, D T_{s}\right)$. (b) Time interval $\left(D T_{s}, t_{2}\right)$. (c) Time interval $\left(t_{2}, T_{s}\right)$.

For the converter to operate in DCM, it is necessary that

$$
\frac{V_{1}}{V_{1}-V_{2}} \geq \frac{D}{1-D}
$$

Thus, the constraint on the value of the duty-cycle is

$$
D \leq \frac{V_{1}}{2 V_{1}-V_{2}}
$$

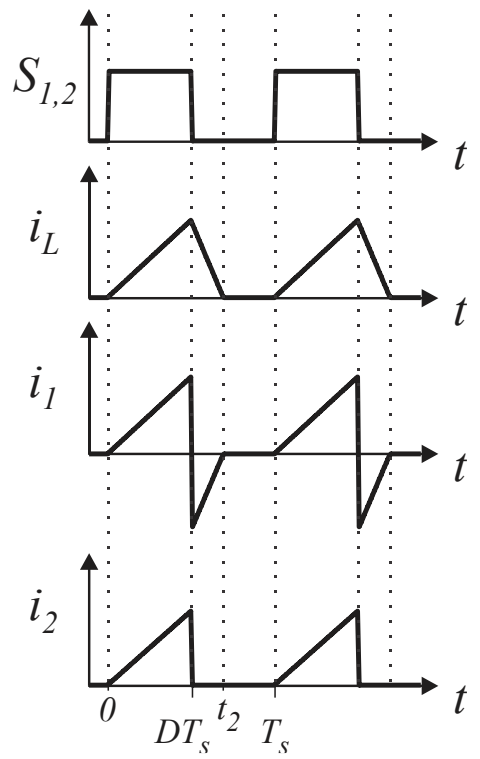

Fig. 9. Typical idealized current waveforms of the converter shown in Fig. 8, for the realization of the SLFR.

The most critical condition occurs when $V_{2}=0$, causing $D \leq 0.5$. Adopting $D=0.5$ the converter must operate in discontinuous conduction mode and this must be the value selected in a practical design.

\section{Numerical Example I}

Consider the circuit shown in Fig. 7, represented by the equivalent circuit shown in Fig. 5, operating in steady-state, with the parameters $V_{1}=100 \mathrm{~V}, C=50 \mu \mathrm{F}, R=50 \Omega, D=0.5$, $L=200 \mu \mathrm{H}$, and $f_{s}=20 \mathrm{kHz}$. The value of the virtual resistance is

$$
R_{v}=\frac{2 L f_{s}}{D^{2}}=\frac{2 \times 200 \times 10^{-6} \times 20 \times 10^{3}}{0.25}=32 \Omega
$$

The output voltage is

$$
V_{2}=\frac{R}{R+R_{v}} \cdot V_{1}=\frac{50}{50+32} \cdot 100=60.97 \mathrm{~V}
$$

The power dissipated in the load resistor $R$ is

$$
P_{2}=\frac{V_{2}^{2}}{R}=\frac{60.97^{2}}{50}=74.34 \mathrm{~W}
$$

The power absorbed by the resistor $R_{v}$ is

$$
P_{v}=\frac{\left(V_{1}-V_{2}\right)^{2}}{R_{v}}=\frac{(100-60.97)^{2}}{32}=47.6 \mathrm{~W}
$$


The current in the power source is

$$
I_{x}=\frac{P_{v}}{V_{1}}=\frac{47.60}{100}=0.476 \mathrm{~A}
$$

The current and power in the source $V_{1}$ are respectively

$$
I_{1}=\frac{V_{1}-V_{2}}{R_{v}}-I_{x}=\frac{100-60.97}{32}-0.476=0.744 \mathrm{~A}
$$

and

$$
P_{1}=V_{1} I_{1}=100 \times 0.744=74.4 \mathrm{~A} .
$$

The input and the output power are equal and the theoretical efficiency is equal to unity since the power absorbed by $R_{v}$ is transferred to the input voltage source $V_{1}$. These numerical results were verified by computer simulation.

\section{TRANSIENT ANALYSIS OF CIRCUITS FORMED BY LOSS-FREE RESISTORS, DC VOLTAGE SOURCES AND CAPACITORS}

\section{A. First RC Circuit}

Consider the circuit shown in Fig. 10, consisting of the voltage source $V_{1}$, the capacitor $C$ and the loss-free resistor $R_{v}$. At $t=0$, the initial charge on the capacitor is $q_{o}=0$.

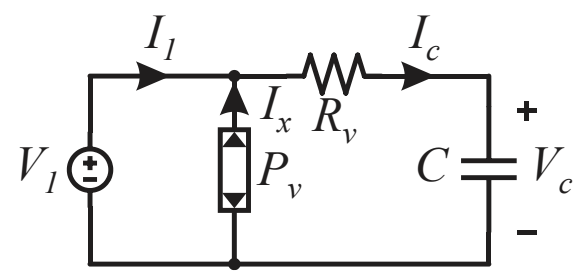

Fig. 10. Circuit formed by a DC voltage source, a capacitor and an SLFR.

The voltage across and the current through the capacitor $C$ are given respectively by

and

$$
V_{c}(t)=V_{1}\left(1-e^{\frac{-t}{\tau}}\right)
$$

$$
I_{c}(t)=\frac{V_{1}}{R_{v}}\left(e^{\frac{-t}{\tau}}\right)
$$

where the time constant is $\tau=R_{v} C$. As expected, in steady-state the voltage across the capacitor is $V_{c}=V_{1}$ and the current $I_{c}=0$, as in the circuit formed by a dissipative resistor.

The current in the power source is given by

$$
I_{x}(t)=\frac{R_{v} I_{c}(t)^{2}}{V_{1}}
$$

Substituting (12) in (13) we find

$$
I_{x}(t)=\frac{V_{1}}{R_{v}} e^{-\frac{2 t}{\tau}}
$$

The current in the voltage source $V_{1}$ is given by

$$
I_{1}(t)=I_{c}(t)-I_{x}(t)
$$

Substitution of (12) and (14) in (15) yields

$$
I_{1}(t)=\frac{V_{1}}{R_{v}} e^{-\frac{t}{\tau}}\left(1-e^{-\frac{2 t}{\tau}}\right)
$$

The instantaneous energy delivered by the voltage source $V_{1}$ is given by

$$
E_{1}(t)=\int V_{1} I_{1}(t) d t
$$

Substitution of (16) in (17) and integration gives

$$
E_{1}(t)=\frac{V_{1}^{2}}{2 R_{v}} \tau\left(e^{-\frac{t}{\tau}}-1\right)^{2}
$$

The total energy delivered by the voltage source $V_{1}$ during the transient time interval is given by

$$
E_{1}=\frac{1}{2} C V_{1}^{2}
$$

which is equal to the energy stored in the capacitor, given by

$$
E_{c}=\frac{1}{2} C V_{1}^{2}
$$

We conclude that employing the loss-free resistor, we can charge a capacitor from a DC voltage source without energy loss. When the capacitor is charged through a conventional resistor, as it is well known, the energy dissipated in the resistor is equal to the value of the energy transferred to the capacitor during the transient time interval. The relevant voltage and current waveforms are plotted in Fig. 11, with $V_{1}=100 \mathrm{~V}, C=100 \mu \mathrm{F}$ and $R_{v}=32 \Omega$. The capacitor voltage, the capacitor current and the input current are denoted respectively by $V_{c a}, i_{c a}$ and $i_{1 a}$ for the circuit with the loss-free resistor, and by $V_{c b}, i_{c b}$ and $i_{1 b}$ for the circuit with a dissipative resistor. 


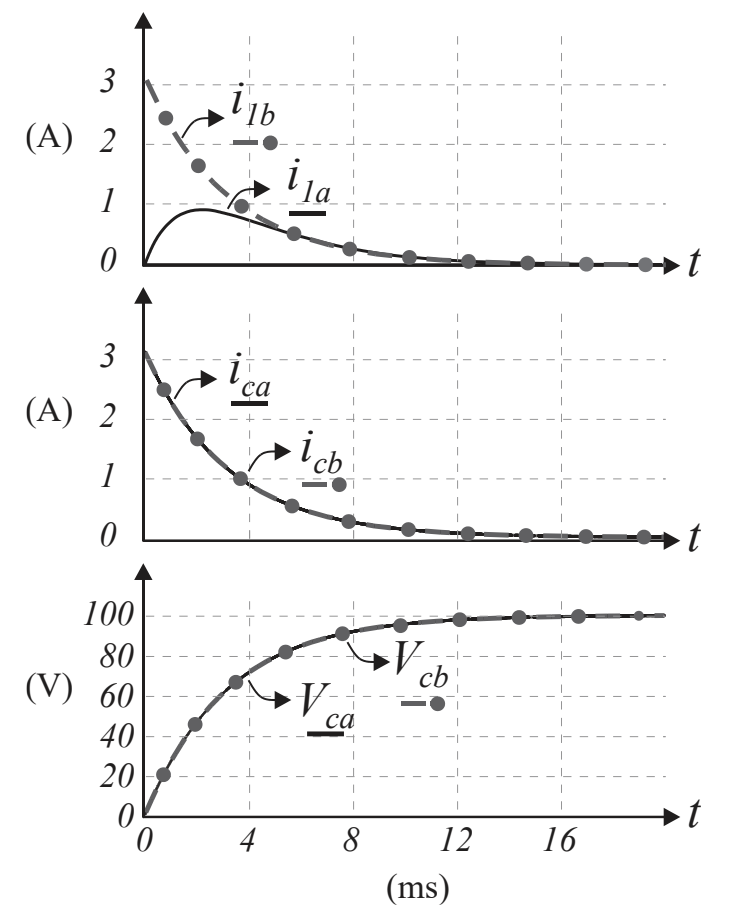

Fig. 11. Simulation results for the circuit shown in Fig. 10. (a) With a loss-free resistor. (b) With a dissipative resistor connected in series with the voltage source $V_{l}$ and the capacitor $C$.

\section{B. Second RC Circuit}

Consider the circuit shown in Fig. 12, consisting of the capacitor $C$, the voltage source $V_{o}$, and the loss-free resistor $R_{v}$. At $t=0$, the initial voltage on the capacitor is $V_{c}(0)=V_{c o}$ and $V_{c o}>V_{o}$.

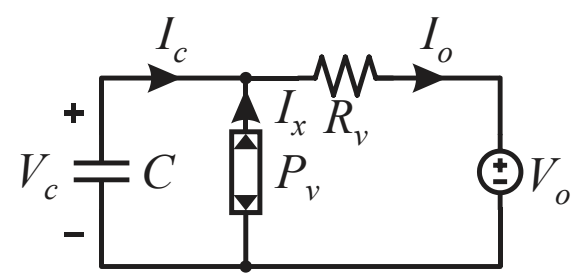

(a)

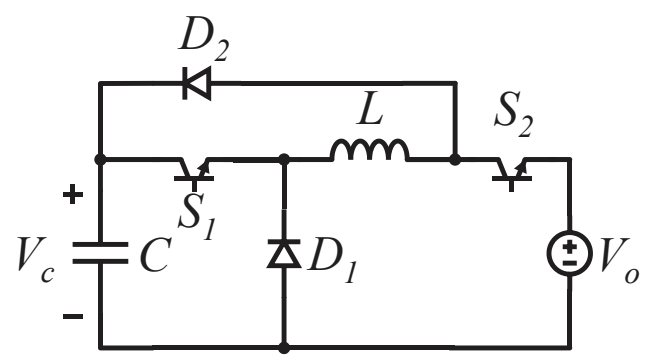

(b)

Fig. 12. (a) Circuit formed by a capacitor, a loss-free resistor and a voltage source. (b) Realization using the topological variation of the positive output buckboost converter operated in DCM.

The current through the voltage source during the discharge of the capacitor is

$$
I_{o}(t)=\frac{V_{c}(t)-V_{o}}{R_{v}}
$$

The power of the power source is given by

$$
P_{v}(t)=\frac{\left(V_{c}(t)-V_{o}\right)^{2}}{R_{v}}
$$

and the respective current is given by

$$
I_{x}(t)=\frac{\left(V_{c}(t)-V_{o}\right)^{2}}{R_{v} V_{c}(t)}
$$

Since the current in the capacitor is

$$
I_{c}(t)=I_{o}(t)-I_{x}(t)
$$

substituting (21) and (23) in (24) we find

$$
I_{c}(t)=\frac{V_{c}(t)-V_{o}}{R_{v}}-\frac{\left(V_{c}(t)-V_{o}\right)^{2}}{R_{v} V_{c}(t)}
$$

The voltage across the capacitor is described by

$$
I_{c}(t)=C \frac{d V_{c}(t)}{d t}
$$

Substituting (25) in (26) and rearranging the terms we find

$$
V_{c}(t) \frac{d V_{c}(t)}{d t}+\frac{V_{o}}{R_{v} C} V_{c}(t)=\frac{V_{o}^{2}}{R_{v} C}
$$

This first-order nonlinear equation (27), that describes the circuit shown in Fig. 12, is an Abel equation of the second kind. From (27) we can find

$$
\left(\frac{V_{c}(t)}{V_{o}-V_{c}(t)}\right) d V_{c}(t)=\frac{V_{o}}{\tau} d t
$$

where $\tau=R C$, from which

$$
\int\left(\frac{V_{c}(t)}{V_{o}-V_{c}(t)}\right) d V_{c}(t)=\int \frac{V_{o}}{\tau} d t+K
$$

Hence,

$$
-V_{c}(t)-V_{o} \ln \left[V_{c}(t)-V_{o}\right]=\frac{V_{o}}{\tau} t+K
$$

To determine $K$, we set $t=0$ in (30) and substitute the initial voltage $V_{c o}$ for $V_{c}$. Hence,

$$
K=-V_{c o}-V_{o} \ln \left(V_{c o}-V_{o}\right)
$$


The explicit solution of the non-linear algebraic equation (30) is only possible employing the Lambert $\mathrm{W}$ function, as follows.

$$
\left.V_{c}(t)=V_{o}+e^{\left[\frac{K}{V_{o}}+\frac{t}{\tau}+\text { Lambert } W\right.}\left(\frac{e^{-\left(1+\frac{K}{V_{o}}+\frac{t}{\tau}\right)}}{V_{o}}\right)+1\right]
$$

The circuits shown in Figs. 12 (a) and 12 (b) were simulated, and the results are shown in Fig. 13. The values for capacitor voltage and current for the two circuits are the same and are denoted by $V_{c a}$ and $I_{c a}$, respectively. The parameters are $V_{o}=100 \mathrm{~V}, \quad C=200 \mu \mathrm{F}, \quad V_{c o}=180 \mathrm{~V}, \quad R_{v}=32 \Omega$, $f_{S}=20 \mathrm{kHz}$, and duty-cycle $D=0.5$. An identical circuit was simulated with a dissipative resistor $R=32 \Omega$ and the corresponding capacitor voltage and current are denoted by $V_{c b}$ and $I_{c b}$. These waveforms demonstrate that the trajectories of the capacitor voltages and currents are not equal.

(V)
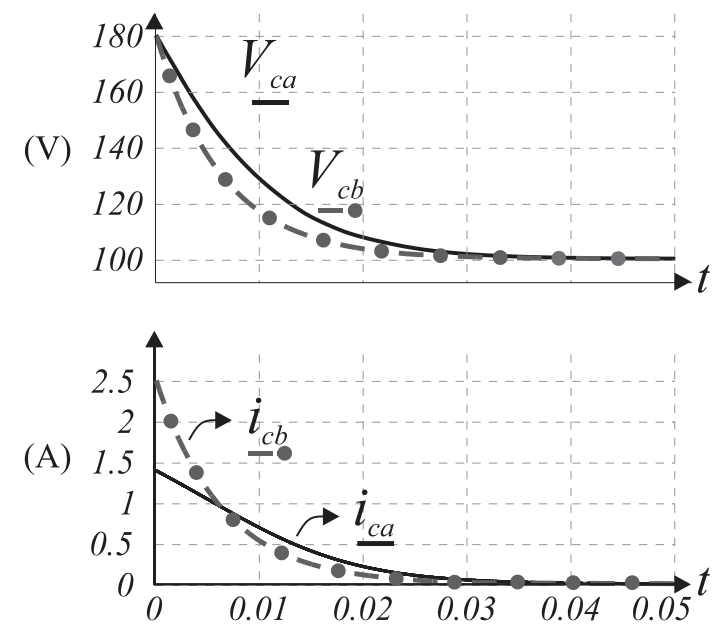

(s)

Fig. 13. Simulation results for the circuit shown in Fig. 12. (a) With a loss-free resistor. (b) With a dissipative resistor connected in series with the capacitor $C$ and the voltage source $V_{o}$.

It can easily be demonstrated that during the discharge of the capacitor through a dissipative resistor, the energy transformed into heat in the resistor is given by

$$
E_{R}=\frac{1}{2} C\left(V_{c o}-V_{o}\right)^{2}
$$

Therefore, when $V_{c o}=2 V_{o}$, half of the energy initially stored in the capacitor is lost in the resistor during the transient time interval. With the use of the loss-free resistor, no energy is lost and all of the energy initially stored in the capacitor is transferred to the voltage source $V_{o}$.

\section{Third RC Circuit}

A circuit formed by two capacitors $C_{1}$ and $C_{2}$ associated in series with the loss-free resistor $R_{v}$ is shown in Fig. 14. At $t=0$, the initial voltages on the capacitors are $V_{c 1 o}$ and $V_{c 2 o}$, respectively, and $V_{c 1 o} \geq V_{c 2 o}$. During the transient period, part of the energy stored in capacitor $C_{1}$ is transferred to capacitor $C_{2}$. The transient period theoretically finishes at the instant when $V_{c 1}=V_{c 2}$. In order to simplify the analysis of the transient behavior of the circuit, it is assumed that $C_{1}=C_{2}=C$.

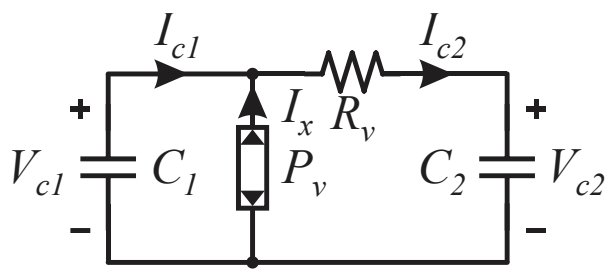

(a)

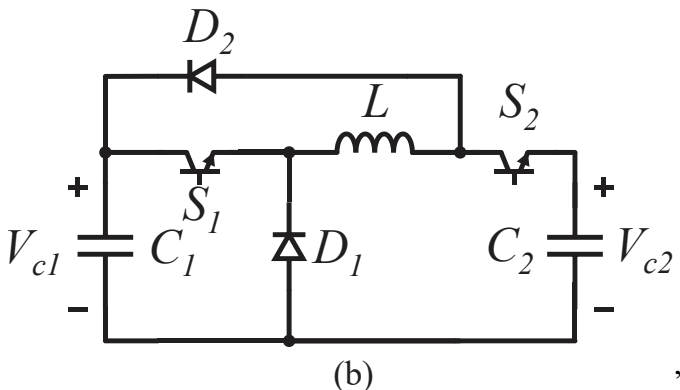

Fig. 14. (a) Circuit formed by the series association of two capacitors and a lossfree resistor. (b) Physical realization using the positive output buck-boost converter operated in DCM

The current in capacitor $C_{2}$ is given by

$$
I_{c 2}(t)=\frac{V_{c 1}(t)-V_{c 2}(t)}{R_{v}}
$$

This current is also described by

$$
I_{c 2}(t)=C \frac{d V_{c 2}(t)}{d t}
$$

Substituting (34) in (35) we find

$$
\frac{d V_{c 2}(t)}{d t}=\frac{1}{R_{v} C}\left[V_{c 1}(t)-V_{c 2}(t)\right]
$$

As follows from Kirchhoff's current law

$$
I_{c 1}(t)=I_{x}(t)-I_{c 2}(t)
$$

The instantaneous power of the power source is given by

$$
P_{v}(t)=\frac{\left[V_{c 1}(t)-V_{c 2}(t)\right]^{2}}{R_{v}}
$$


Hence,

$$
I_{x}(t)=\frac{\left[V_{c 1}(t)-V_{c 2}(t)\right]^{2}}{R_{v} V_{c 1}(t)}
$$

Substitution of (35) and (39) in (37) yields

$$
I_{c 1}(t)=\frac{\left[V_{c 1}(t)-V_{c 2}(t)\right]^{2}}{R_{v} V_{c 1}(t)}-\frac{V_{c 1}(t)-V_{c 2}(t)}{R_{v}}
$$

The current in capacitor $C_{1}$ is described by

$$
I_{c 1}(t)=C \frac{d V_{c 1}(t)}{d t}
$$

Substituting (40) in (41) and rearranging the terms, we find

$$
\frac{d V_{c 1}(t)}{d t}=-\frac{1}{R_{v} C} \frac{V_{c 2}(t)}{V_{c 1}(t)}\left[V_{c 1}(t)-V_{c 2}(t)\right]
$$

The two first-order nonlinear differential equation system (36) and (42) describe the transient behavior of the circuit of Fig. 14(a). There is no explicit analytical solution for this system of equations, despite the apparent simplicity of the circuit they describe.

Dividing (42) by (36) and rearranging the terms we find

$$
V_{c 1}(t) d V_{c 1}(t)+V_{c 2}(t) d V_{c 2}(t)=0
$$

Integration of (43) yields

$$
V_{c 1}(t)^{2}+V_{c 2}(t)^{2}=K
$$

To determine the constant $K$ we set $t=0$ in (44) and substitute the initial voltages $V_{c 10}$ and $V_{c 2 o}$. Hence

$$
K=V_{c 10}^{2}+V_{c 2 o}^{2}
$$

Expressions (44) and (45) express the principle of energy conservation and this is a consequence of the non-dissipation of energy in the series loss-free resistor $R_{v}$.

From (44) we obtain

$$
V_{c 1}(t)=\sqrt{K-V_{c 2}(t)^{2}}
$$

Substitution of (46) in (36) yields

$$
\frac{d V_{c 2}(t)}{d t}=\frac{1}{R_{v} C}\left[\sqrt{K-V_{c 2}(t)^{2}}-V_{c 2}(t)\right]
$$

which is not integrable. As a consequence, there is no exact and explicit solution for the differential equation (47).

Making $\frac{d V_{c 2}(t)}{d t}=0$ in (47), we find the final value of the voltage across capacitor $C_{2}$, which equal to the final voltage of capacitor $C_{1}$, given by

$$
V_{c f}=\sqrt{\frac{V_{c 1 o}^{2}+V_{c 2 o}^{2}}{2}}
$$

For the particular case in which $V_{c 2 o}=0$, the final voltages on the capacitors is

$$
V_{c f}=\frac{V_{c l o}}{\sqrt{2}}
$$

Expression (44) shows that the trajectory described when $V_{c 1}(t)$ is plotted against $V_{c 2}(t)$ is circular, with the coordinates of the center at the origin and with radius $r=\sqrt{K}$, which is plotted in Fig. 15.

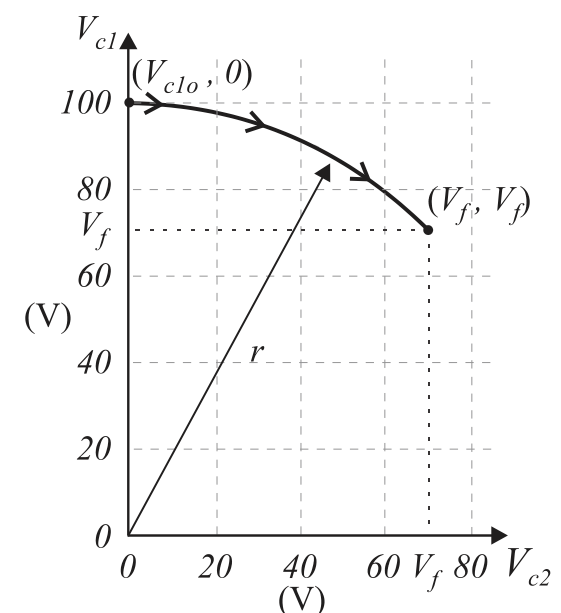

Fig. 15. State space trajectory described by plotting voltage $V_{c 1}$ against $V_{c 2}$ for the circuit shown in Fig. 14, with $V_{c 1 o}=100 \mathrm{~V}$ and $V_{c 2 o}=0 \mathrm{~V}$.

An approximate solution of the differential equation (47) in the form of an analytic expression is

$$
V_{c 2}(t)=V_{f}\left(1-e^{\frac{-\alpha t}{\tau}}\right)
$$

where $\tau=R_{v} C$ and $\alpha$ is a constant to be determined.

Differentiating (5) with respect to time we find

$$
\frac{d V_{c 2}(t)}{d t}=\frac{V_{f} \alpha}{\tau}\left(1-e^{\frac{-\alpha t}{\tau}}\right)
$$

Substitution of (50) and (51) in (46) yields

$$
\alpha V_{f} e^{\frac{-\alpha t}{\tau}}=\left[\sqrt{K-V_{f}^{2}\left(1-e^{-\frac{\alpha t}{\tau}}\right)^{2}}-V_{f}\left(1-e^{-\frac{\alpha t}{\tau}}\right)\right]
$$

To determine the constant $\alpha$ we set $t=0$ in (52) and find $\alpha=\sqrt{2}$, which substituted in (46) and (50) gives 


$$
V_{c 1}(t)=\frac{1}{\tau} \sqrt{K-V_{f}^{2}\left(1-e^{\frac{-\sqrt{2} t}{\tau}}\right)^{2}}
$$

and

$$
V_{c 2}(t)=V_{f}\left(1-e^{\frac{-\sqrt{2} t}{\tau}}\right)
$$

which describe the transient voltages across the capacitors $C_{1}$ and $C_{2}$.

Since the energy is transferred from capacitor $C_{1}$ to capacitor $C_{2}$ through a loss-free resistor, all of the energy decrease in capacitor $C_{1}$ is transferred to capacitor $C_{2}$ and no energy is lost by the circuit during the transient time interval. In the case where the energy exchange takes place through a dissipative resistor, only half of the energy initially stored in capacitor $C_{1}$ is transferred to capacitor $C_{2}$, while the other half is transferred to the resistor and lost in the form of heat.

\section{Numerical Example II}

Consider the circuits shown in Fig. 14 with the parameters $C_{1}=C_{2}=C=500 \mu \mathrm{F}, V_{c 1 o}=100 \mathrm{~V}, V_{c 2 o}=0 \mathrm{~V}, f_{s}=20 \mathrm{kHz}$, $D=0.5$ and $L=200 \mu \mathrm{H}$. The value of the series loss-free resistance is

$$
R_{v}=\frac{2 L f_{s}}{D^{2}}=\frac{2 \times 200 \times 10^{-6} \times 20 \times 10^{3}}{0.25}=32 \Omega
$$

The voltage across the capacitors in the steady-state is

$$
V_{f}=\frac{V_{c 1 o}}{\sqrt{2}}=\frac{100}{\sqrt{2}}=70.71 \mathrm{~V}
$$

The time constant is $\tau=R_{v} C=32 \times 500 \times 10^{-6}=0.016 \mathrm{~s}$ and $\alpha=\sqrt{2}=1.414$. The transient voltages across the capacitors $C_{1}$ and $C_{2}$ are respectively

$$
V_{c 1}(t)=\sqrt{100^{2}-70.71^{2}\left(1-e^{-108.25 t}\right)^{2}}
$$

and

$$
V_{c 2}(t)=70.71\left(1-e^{-108.25 t}\right)
$$

The transient voltages across the capacitors $C_{1}$ and $C_{2}$, generated by the computer simulations of the circuits shown in Fig. 14 are plotted in Fig. 16. It is well known that during the transient transfer of energy between two identical capacitors, with one initially discharged, half of the energy stored in the initially charged capacitor is dissipated in the resistor, regardless of the value of that resistor. On the other hand, this process occurs theoretically without losses with the use of the loss-free resistor and this can be desirable in many practical applications.

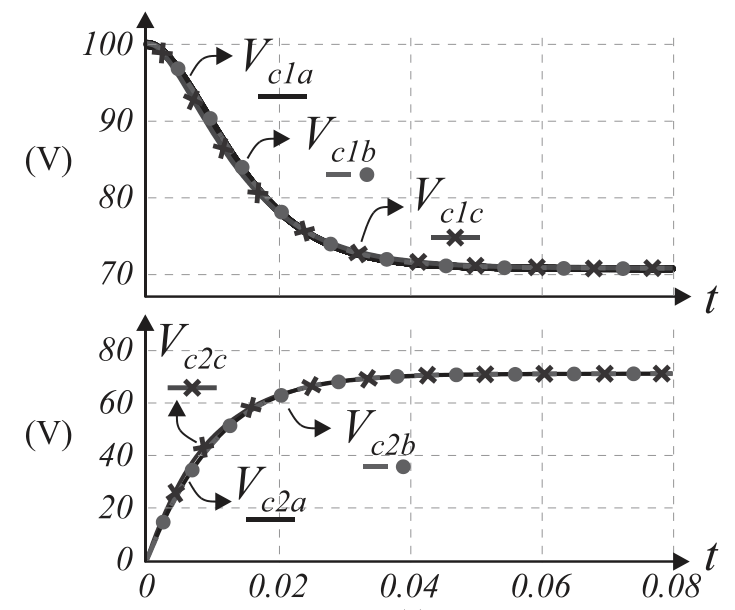

(s)

Fig. 16. Transient voltages across the capacitors $C_{1}$ and $C_{2}$. (a) Simulation of the equivalent circuit shown in Fig. 14(a). (b) Simulation of the circuit shown in Fig. 14(b). (c) Generated by equations (55) and (56), with $\alpha=\sqrt{3}$.

\section{APPLICATION OF THE SLFR IN REGENERATIVE VOLTAGE CLAMPING CIRCUIT}

In addition to the applications mentioned in the previous section, many others can benefit from the use of the SLFR. Below we describe a possible practical application, by way of illustration.

Fig. 17 (a) shows the power stage diagram of current-fed pushpull dc-dc converter, which is suitable for applications with high input current and high output voltage [10]. A recognized drawback of this topology is the energy trapped in the transformer leakage inductances. This can subject the power semiconductors to excessive voltages and also compromise the converter efficiency. Aiming to protect the power switches, a voltage clamping circuit is commonly used, which is formed by the diodes $D_{c 1}$ and $D_{c 2}$, the capacitor $C_{c}$, and the resistor $R_{c}$. The transformer leakage inductances are denoted by $L_{l 1}$ and $L_{l 2}$. The energy stored in the leakage inductors is transferred to the clamping capacitor and part of this energy is then transferred to the input voltage source $V_{1}$. However, the energy dissipated in the resistor $R_{c}$ can be large and limit the processed power and the switching frequency, required to prevent low efficiency.

In Fig. 17 (b), the conventional dissipative resistor is replaced with SLFR. Thus, theoretically, all of the energy trapped in the leakage inductances is recycled to the input voltage source, without losses, enabling operation with higher power and frequency, without sacrificing the converter efficiency. The lossfree resistor is realized using the positive output buck-boost converter operating in DCM shown in Fig. 14(b).

To verify the impact of the voltage clamping circuit with the SLFR, a converter was simulated with the parameters $V_{1}=100 \mathrm{~V}, \quad L_{1}=200 \mu \mathrm{H}, \quad R_{c}=50 \Omega, \quad C_{c}=4.7 \mu \mathrm{F}$, magnetizing inductance $L_{m}=500 \mu \mathrm{H}$, leakage inductances $L_{l}=0.5 \mu \mathrm{H}, C_{o}=20 \mu \mathrm{F}, R_{o}=200 \Omega$, switching frequency $f_{s}=30 \mathrm{kHz}$, transformer turns-ration $a=5$ and duty-cycle $D=0.5$. The parameters of the power stage that realizes the SLFR in Fig. 17 (b) are $L_{2}=208 \mu \mathrm{H}, f_{s 2}=30 \mathrm{kHz}$ and dutycycle $D_{2}=0.5$. 


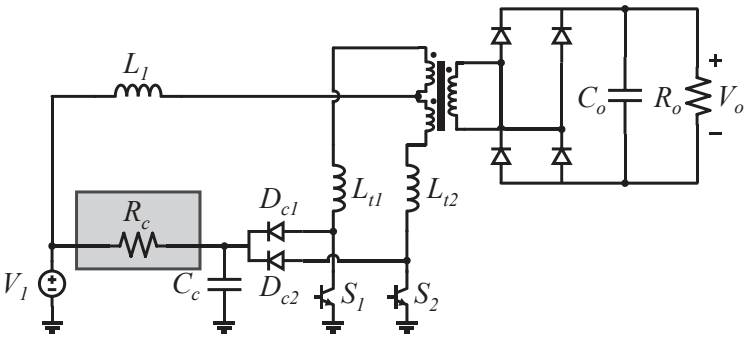

(a)

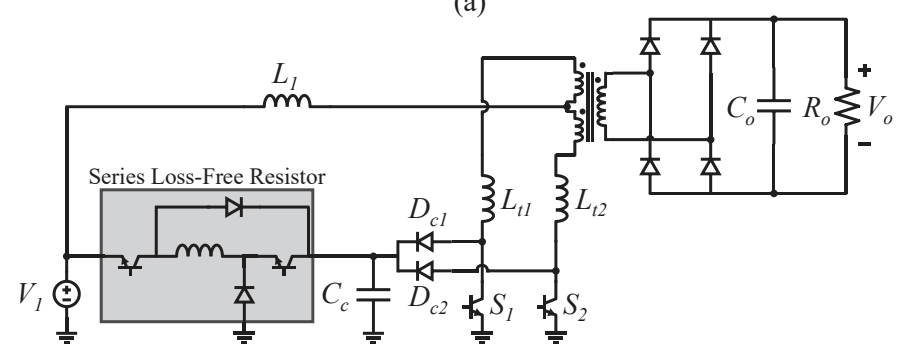

(b)

Fig. 17. Current-fed push-pull dc-dc converter. (a) With dissipative voltage clamping circuit. (b) With non-dissipative voltage clamping circuit, using a series loss-free resistor.

All components are assumed ideal. The simulation results are shown in Table I. It can be noted that the energy loss in the conventional voltage clamping circuit is very high and that it is practically canceled with the use of the SLFR.

TABLE I

SiMUlation RESUltS FOR THE CONVERTERS SHOWN IN FIG. 17

\begin{tabular}{lll}
\hline \hline Parameter & $\begin{array}{l}\text { Dissipative } \\
\text { Resistor }\end{array}$ & SLFR \\
\hline Output voltage $\left(V_{o}\right)$ & $472 \mathrm{~V}$ & $473 \mathrm{~V}$ \\
Output power $\left(P_{o}\right)$ & $1114 \mathrm{~W}$ & $1119 \mathrm{~W}$ \\
Capacitor Voltage $\left(V_{c}\right)$ & $196.3 \mathrm{~V}$ & $211.32 \mathrm{~V}$ \\
Resistor current $\left(I_{R_{C}}\right)$ & $2.97 \mathrm{~A}$ & $3.27 \mathrm{~A}$ \\
Power dissipated in the resistor $\left(P_{R_{C}}\right)$ & $441 \mathrm{~W}$ & 0 \\
Input current $\left(I_{1}\right)$ & $33.4 \mathrm{~A}$ & $23.33 \mathrm{~A}$ \\
Input power $\left(P_{1}\right)$ & $1556 \mathrm{~W}$ & $1120 \mathrm{~W}$ \\
Efficiency $(\eta)$ & $72 \%$ & $100 \%$ \\
\hline \hline
\end{tabular}

The authors of [10] propose the use of the current-fed pushpull converter as a preamplifier stage of a constant current charger for high-voltage capacitors and a novel snubber technique for this converter family was introduced, to limit the voltage across the power semiconductors and reduce the commutation losses. In [11], the use of the active-clamping technique is proposed to suppress the voltage spikes across the primary power semiconductors and to recycle the energy stored in the transformer leakage inductances. The solutions proposed in [10-11] need synchronization between the gate-drive signals of the main power switches, and the additional switches, with appropriate dead-times between them. The use of the SLFR does not require synchronization and it can be treated as a resistor in the circuit, which simplifies the analysis and the selection of the parameters. Thus, the SLFR and may be a suitable candidate for this application.

\section{CONCLUSION}

The transient behavior of DC networks consisting of voltage sources, capacitors, and series loss-free resistors (SLFRs) was analyzed. The SLFR was realized by means of a topological variation of the positive output voltage buck-boost converter operated in discontinuous conduction mode (DCM). It is demonstrated that even simple RC circuits containing the SLFR exhibit behavior described by Abel non-linear differential equations that do not have exact analytical solution.

Some possible applications of the SLFR in power electronics, DC microgrids and renewable energy power systems, include the loss-free charging and discharging of capacitor banks, voltage equalization of capacitors, and loss-free voltage clamping circuits for current-fed push-pull converters.

The concept of SLFR can also be used for voltage control in DC microgrids by means of the droop voltage technique, equalization of power and current in voltage sources associated in parallel (which includes batteries and DC-DC converters), and also input and output voltage natural balance in input-series output-series (ISOS) association of DC converters.

\section{ACKNOWLEDGMENT}

The work of Kaio C. M. Nascimento, graduate student in electrical engineering with Federal University of Santa Catarina (UFSC), who collaborated in the manuscript editing, is gratefully acknowledged.

\section{REFERENCES}

[1] S. Singer, "Realization of loss-free resistive elements," in IEEE Transactions on Circuits and Systems, vol. 37, no. 1, pp. 54-60, Jan. 1990, doi: 10.1109/31.45691.

[2] S. Singer, "The application of 'loss-free resistors' in power processing circuits," 20th Annual IEEE Power Electronics Specialists Conference, Milwaukee, WI, USA, 1989, pp. 843846 vol.2, doi: 10.1109/PESC.1989.48568.

[3] S. Singer and R. W. Erickson, "Canonical modeling of power processing circuits based on the POPI concept," in IEEE Transactions on Power Electronics, vol. 7, no. 1, pp. 37-43, Jan. 1992, doi: 10.1109/63.124575.

[4] S. Singer, S. Ozeri and D. Shmilovitz, "A pure realization of loss-free resistor," in IEEE Transactions on Circuits and Systems I: Regular Papers, vol. 51, no. 8, pp. 1639-1647, Aug. 2004, doi: 10.1109/TCSI.2004.832751.

[5] A. Wang, Hongyi Yin and Shi Wang, "Realization of source with internal loss-free resistive characteristic," in IEEE Transactions on Circuits and Systems I: Fundamental Theory and Applications, vol. 48, no. 7, pp. 830-839, July 2001, doi: 10.1109/81.933324.

[6] A. Cid-Pastor, L. Martinez-Salamero, A. El Aroudi, R. Giral, J. Calvente and R. Leyva, "Synthesis of loss-free resistors based on sliding-mode control and its applications in power processing", Control Eng. Pract., vol. 21, no. 5, pp. 689-699, 2013.

[7] N. Rathore, D. Fulwani, A. K. Rathore and A. R. Gautam, "Adaptive Sliding Mode Based Loss-Free Resistor for PowerFactor Correction Application," in IEEE Transactions on Industry Applications, vol. 55, no. 4, pp. 4332-4343, July-Aug. 2019, doi: 10.1109/TIA.2019.2912799.

[8] N. Rathore, S. Gangavarapu, A. K. Rathore and D. Fulwani, "Emulation of Loss Free Resistor for Single-Stage Three-Phase PFC Converter in Electric Vehicle Charging Application," in IEEE Transactions on Transportation Electrification, vol. 6, no. 1, pp. 334-345, March 2020, doi: 10.1109/TTE.2020.2976878.

[9] S. Singer, R. Giral, J. Calvente, R. Leyva, L. MartinezSalamero and D. Naunin, "Maximum power point tracker based on a loss-free resistor topology", Proc. 5th Eur. Space Power Conf., pp. 65-70, 1998-Sept.21-25. 
[10] T. Weinert, W. Oberschelp and G. Schroder, "A current-fed $\mathrm{DC} / \mathrm{DC}$ converter for the efficient charging of $\mathrm{HV}$ capacitors in mobile applications," 2016 18th European Conference on Power Electronics and Applications (EPE'16 ECCE Europe), Karlsruhe, 2016, pp. 1-10, doi: 10.1109/EPE.2016.7695412.

[11] Q. Wu, Q. Wang, J. Xu and Z. Xu, "Active-clamped ZVS current-fed push-pull isolated $\mathrm{dc} / \mathrm{dc}$ converter for renewable energy conversion applications," in IET Power Electronics, vol. 11, no. 2, pp. 373-381, 202 2018, doi: 10.1049/ietpel.2017.0144. 